\title{
Treating cutaneous squamous cell carcinoma using 5-aminolevulinic acid polylactic-co-glycolic acid nanoparticle-mediated photodynamic therapy in a mouse model
}

\author{
This article was published in the following Dove Press journal: \\ International Journal of Nanomedicine \\ 6 January 2015 \\ Number of times this article has been viewed
}

\section{Xiaojie Wang ${ }^{1,2, *}$ \\ Lei $\mathrm{Shi}^{2}$,* \\ Qingfeng Tu ${ }^{2}$ \\ Hongwei Wang ${ }^{3}$ \\ Haiyan Zhang ${ }^{2}$ \\ Peiru Wang ${ }^{2}$ \\ Linglin Zhang ${ }^{2}$ \\ Zheng Huang ${ }^{4}$ \\ Feng Zhao ${ }^{5}$ \\ Hansen Luan ${ }^{5}$ \\ Xiuli Wang ${ }^{2}$}

'Shanghai Skin Diseases Clinical College of Anhui Medical University,

${ }^{2}$ Shanghai Skin Disease Hospital,

${ }^{3}$ Huadong Hospital, Fudan University,

Shanghai, ${ }^{4}$ MOE Key Laboratory

of OptoElectronic Science and

Technology for Medicine, Fujian

Normal University, Fuzhou, ${ }^{5}$ National

Pharmaceutical Engineering Research

Center, China State Institute of

Pharmaceutical Industry, Shanghai,

People's Republic of China

*These authors contributed equally to this study
Correspondence: Xiuli Wang Shanghai Skin Disease Hospital, I 278 Baode Road, Shanghai 200443, People's Republic of China

Tel +862161833004

Fax +86 2161833021

Email xlwang200I@aliyun.com
Background: Squamous cell carcinoma (SCC) is a common skin cancer, and its treatment is still difficult. The aim of this study was to evaluate the effectiveness of nanoparticle (NP)-assisted 5-aminolevulinic acid (ALA) delivery for topical photodynamic therapy (PDT) of cutaneous SCC.

Materials and methods: Ultraviolet-induced cutaneous SCCs were established in hairless mice. ALA-loaded polylactic-co-glycolic acid (PLGA) NPs were prepared and characterized. The kinetics of ALA PLGA NP-induced protoporphyrin IX fluorescence in SCCs, therapeutic efficacy of ALA NP-mediated PDT, and immune responses were examined.

Results: PLGA NPs enhanced protoporphyrin IX production in SCC. ALA PLGA NPmediated topical PDT was more effective than free ALA of the same concentration in treating cutaneous SCC.

Conclusion: PLGA NPs provide a promising strategy for delivering ALA in topical PDT of cutaneous SCC.

Keywords: 5-aminolevulinic acid (ALA), polylactic-co-glycolic acid (PLGA), nanoparticles (NPs), cutaneous squamous cell carcinoma (SCC), photodynamic therapy (PDT), microneedling

\section{Introduction}

For site-specific and large areas of cutaneous squamous cell carcinoma (SCC), the efficacy of traditional treatment, including surgery, is unsatisfactory. Photodynamic therapy (PDT) might be a potential alternative for such lesions. ${ }^{1}$ Topical PDT mediated by the local application of prodrug 5-aminolevulinic acid (ALA) is a simple, effective, and safe modality for precancerous lesions and carcinoma in situ of the skin. It might be particularly suitable for large and multifocal lesions located on the face and external genital area. ${ }^{2}$ Although ALA PDT shows some promise for the treatment of superficial $\mathrm{SCC},{ }^{3}$ it is not currently recommended for the treatment of invasive SCC, mainly due to the limited bioavailability of ALA in the skin tissue., ${ }^{4,5}$

Nanoparticles (NPs) might be a useful approach for delivering ALA in topical PDT, since NPs might improve ALA stability and cutaneous penetration. ${ }^{6}$ An NP-delivery system might also improve the selective accumulation of photosensitizer in the diseased tissue. ${ }^{7}$ Our in vitro study suggested that the use of polylactic-co-glycolic acid (PLGA) NPs could improve ALA delivery in human cutaneous SCC cells. ${ }^{8}$ The aim of this in vivo study was to evaluate the feasibility of ALA PLGA NP-mediated PDT for the treatment of cutaneous SCC in a mouse model. 


\section{Materials and methods Chemicals}

ALA hydrochloride powder was obtained from Shanghai Fudan-Zhangjiang Bio-Pharmaceutical (Shanghai, People's Republic of China [PRC]). ALA-loaded PLGA NPs (ALA PLGA NPs) were prepared using a modified double-emulsion solvent-evaporation method as previously described. ${ }^{8}$ Encapsulation efficiency was $65.8 \% \pm 7.2 \%$, and ALA-loading capacity was $0.62 \% \pm 0.27 \%$. Sheep antimouse monoclonal antibody against CD4 and CD8, peroxidase-conjugated rabbit antisheep IgG, a Strept Avidin Biotin Complex kit, and 3,3'-diaminobenzidine (DAB) chromogen were obtained from Boster Biological Technology (Wuhan, PRC).

\section{Animal model}

Male SKH-1 hairless mice (6-8 weeks old, Jackson Laboratories) free of skin injuries were housed at $24^{\circ} \mathrm{C}-26^{\circ} \mathrm{C}$ and exposed to daily 12-hour:12-hour light:dark cycles (lights on at $6 \mathrm{am}$ ), with free access to standard mouse chow and tap water. Cutaneous SCCs on the backs of mice were induced by solar-simulated ultraviolet (UV) irradiation (Sigma, Shanghai, PRC) as described before. ${ }^{9}$ Specifically, the mice in experimental groups were irradiated with the minimal erythema dose (UVB $240 \mathrm{~mJ} \cdot \mathrm{cm}^{-2} \cdot \mathrm{d}^{-1}$ and UVA $2,160 \mathrm{~mJ} \cdot \mathrm{cm}^{-2} \cdot \mathrm{d}^{-1}$ ) for 5 consecutive days weekly. Irradiation was stopped when papules measured equal to or more than $1 \mathrm{~mm}$ in diameter for 2 consecutive weeks. The number of tumors $>1 \mathrm{~mm}$ in diameter was counted. Histopathological examination was carried out to observe the histological changes of skin lesions. Criteria for histopathological diagnosis of SCC were 1) neoplasm, 2) squamous epithelial, 3) nuclear characteristics (eg, crowded, pleomorphic, large, and often heterochromatic), and 4) cytoplasmic characteristics, including eosinophilic and abnormal signs of cornification (eg, dyskeratotic cells, parakeratosis) atop surface epidermis or in infundibular epidermis and/or aggregations of neoplastic cells (horn pearls). ${ }^{10}$

\section{Preparation of ALA cream}

Lyophilized powder of ALA PLGA NPs, ALA hydrochloride powder, and oil-in-water matrix cream were weighed. An hour before use, ALA hydrochloride powder and lyophilized ALA PLGA NP powder were mixed with oil-in-water matrix cream to make $0.8 \%$ ALA cream or ALA PLGA NP cream containing $0.8 \%$ ALA.

\section{Measurement of PplX fluorescence}

A total of six tumor-bearing mice were divided into two groups (three mice/group). Fresh ALA PLGA NP cream or ALA cream was topically applied onto the tumors. The relative fluorescence intensity of ALA-induced protoporphyrin IX (PpIX) in the tumor was assessed at predetermined time points between 1 and 9 hours using a spectrometer (LFL-Curalux; provided by the Laser Institute of LudwigMaximilians-Universität, Munich, Germany) in the dark. The excitation wavelength was set at $405 \mathrm{~nm}$, and the fluorescence-emission wavelength at $635 \mathrm{~nm}$. Fluorescence images were also taken using a Woods lamp and digital camera equipped with UV filter.

\section{PDT treatment}

A total of 24 tumor-bearing mice were randomly divided into four groups (six mice/group). Ten visible tumors of similar size were selected from each group. The volume of each tumor ranged between 61.60 and $96.28 \mathrm{~mm}^{3}$. ALA PLGA NP cream (ALA PLGA NP PDT group) and ALA cream (ALA PDT group) were topically applied onto tumor surfaces for appropriate times (according to the fluorescence curve). Tumors not treated were used as controls (control group). Mice were irradiated by a helium-neon laser $(632.8 \mathrm{~nm}$; Shanghai Laser Research Institute, Shanghai, PRC) at a power density of $8.6 \mathrm{~mW} / \mathrm{cm}^{2}$ and energy density of $15 \mathrm{~J} / \mathrm{cm}^{2}$. After treatment, mice were kept in the dark for 24 hours. PDT treatment was repeated once a week for 4 weeks. The mice were observed daily, and the tumors were measured every week for 2 months. Mice with a maximum tumor volume $>500 \mathrm{~mm}^{3}$ were killed by cervical dislocation.

\section{Immunohistochemical studies}

To study immunological responses, a total of 27 mice were randomly divided into three treatment groups, and nine mice were randomly divided into the control group. At determined time points (before treatment, after 1 day, 3 days, and 7 days of treatment, three mice/time point), mice were killed and one tumor located in the center was excised. Paraffinembedded tumors were sectionally cut $(5 \mu \mathrm{m})$, and three slides from each tumor were first rehydrated and treated with antigen-retrieval citrate buffer. They were then stained with antibodies against CD4 and CD8, followed by peroxidaseconjugated rabbit antisheep IgG. Tissue sections were stained using a DAB chromogen and hematoxylin counterstain and observed under a light microscope. The positive cells were counted in five randomly selected high-power fields (400×) on each slide. The results are expressed as means \pm standard deviation (positive cells/field/slice).

\section{Combination of microneedling}

Tumor-bearing mice were divided into three different treatment groups (three mice/group). ALA PLGA NP cream or 
ALA cream were topically applied on the tumors on their backs. The third group was treated by ALA PLGA NPs with a combination of microneedling. The roller (manufactured by Clinical Resolution Laboratory) was passed back and forth for an average of ten times in one direction before changing it. The roller utilized for this study was $3 \mathrm{~mm}$ in depth. Immediately after "needling", ALA PLGA NP cream was applied on the tumor. The fluorescence intensity of ALA-induced PpIX was assessed using the LFL-Curalux spectrometer, as described earlier.

\section{Determination of cellular distribution of ALA PLGA NPs}

A total of nine tumor-bearing mice were divided into three different treatment groups (three mice/group). ALA PLGA NPs were applied to tumors with or without pretreatment of microneedling. Mice not treated were controls. After 3 hours of incubation, mice were killed and their tumors excised and cut into $1 \mathrm{~mm}^{3}$ lumps. The lump was fixed with $3 \%$ glutaraldehyde, refixed with $1 \%$ osmium tetroxide, and dehydrated with acetone. Tumor lumps were cut into $70 \mathrm{~nm}$ superthin sections, double-stained with uranyl acetate and lead citrate, and observed under a transmission electron microscope (JEM-1230, 80 kV; JEOL, Tokyo, Japan). ALA NPs in individual cells were counted.

\section{Statistical analysis}

All experiments were repeated at least three times. SPSS 13.0 software (IBM Corporation, Armonk, NY, USA) was used to perform statistical tests. Two-way analysis of variance was used to compare the time course of PpIX generation by ALA PLGA NPs and $0.8 \%$ ALA and also the tumor size between groups. Independent $t$-tests were used to compare the PpIX fluorescence at a selected time point and also the tumor size of different groups. $P<0.05$ was considered statistically significant.

\section{Results \\ Features of cutaneous SCCs}

After 4 months of continuous UV irradiation, needle-size papules started to appear, and gradually grew to various sizes of cauliflower-type lesions. Pathological examination revealed characteristic pathological changes of SCCs. Lesions between 1 and $3 \mathrm{~mm}$ thickness were mainly SCC in situ, and those thicker than $4 \mathrm{~mm}$ tended to be invasive (Figure 1C and D), and normal skin is shown in Figure 1A and $\mathrm{B}$ as a control.

\section{PpIX-fluorescence kinetics}

After topical application of ALA PLGA NP cream (equivalent to $0.8 \%$ ALA), PpIX-fluorescence intensity increased

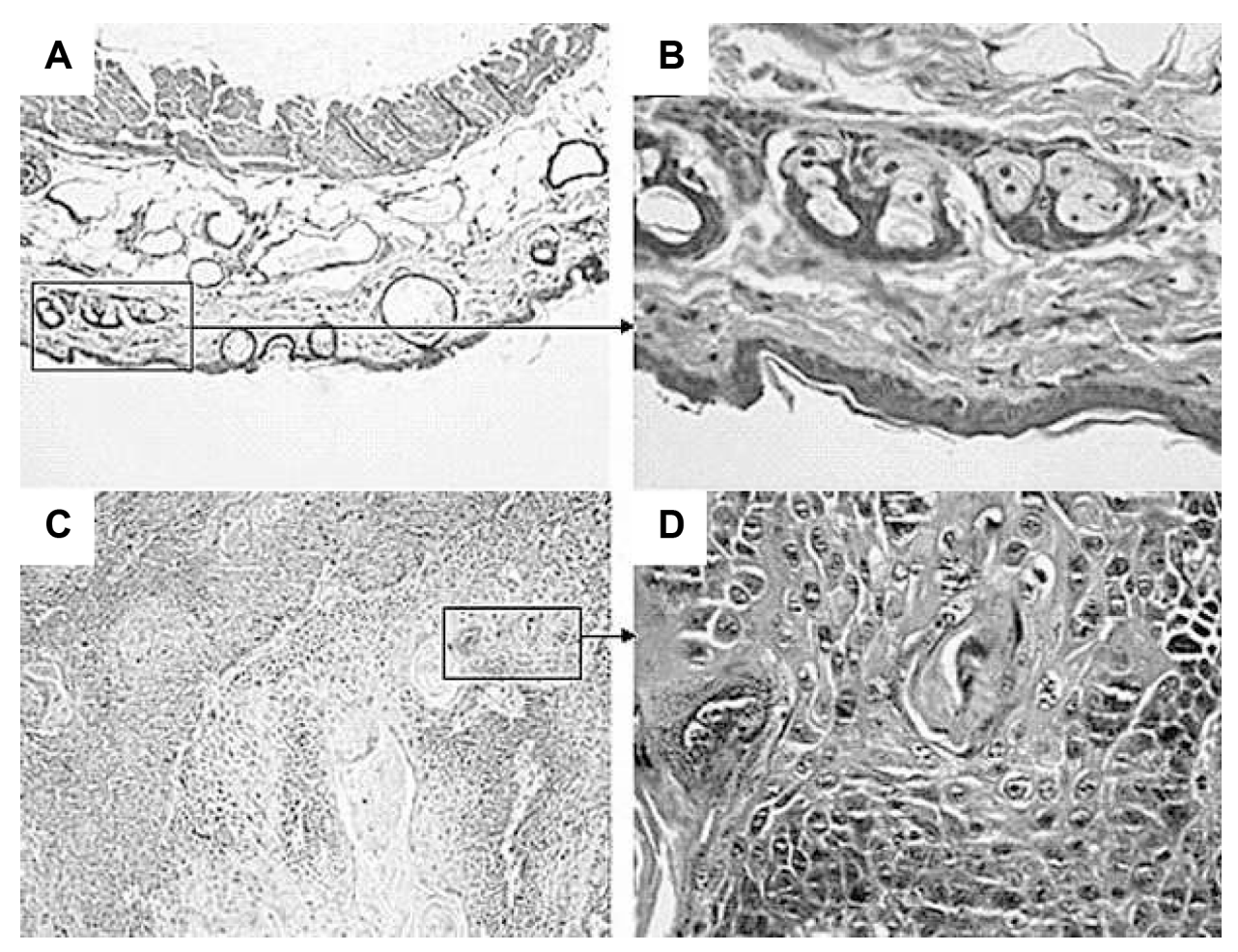

Figure I Histopathological examination of normal and cancerous skin.

Notes: The normal skin showing clear and complete structures: (A) 100× and (B) 400×. Squamous cell carcinoma tissue showing a large number of atypia cells, pathological mitotic, and keratosis beads: (C) $100 \times$ and (D) $400 \times$. 


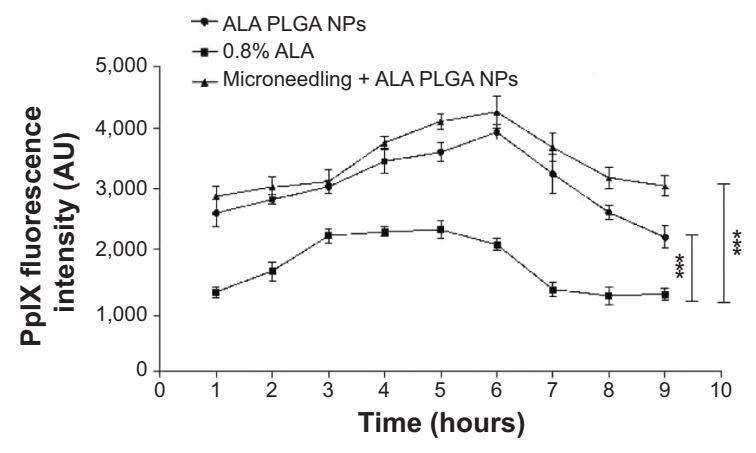

Figure 2 Measurement of PpIX fluorescence in SCC lesions.

Notes: ${ }^{* * *} p<0.000 \mathrm{I}$. Each point represents the mean \pm standard deviation $(n=3)$. Abbreviations: PpIX, protoporphyrin IX; ALA, 5-aminolevulinic acid; PLGA, polylactic-co-glycolic acid; NPs, nanoparticles; SCC, squamous cell carcinoma.

during 1-6 hours and reached a peak at 6 hours before beginning to decline. After treatment with ALA cream of the same concentration, PpIX-fluorescence intensity reached the peak level at 3 hours, before beginning to decline at 6 hours. Although the release of ALA from NPs might have been slower, the relative PpIX-fluorescence intensity of the ALA PLGA NP group was higher than that of the ALA group, as shown in Figure 2.

\section{PplX-fluorescence imaging}

In situ fluorescence-image examination revealed that compared to the $0.8 \%$ ALA group (Figure 3A), the fluorescence of the ALA PLGA NP group (Figure 3B) was stronger and more confined to the tumor site, and the fluorescence intensity in surrounding tissues was weaker. This suggested that ALA PLGA NPs were highly selective for targeting SCCs.

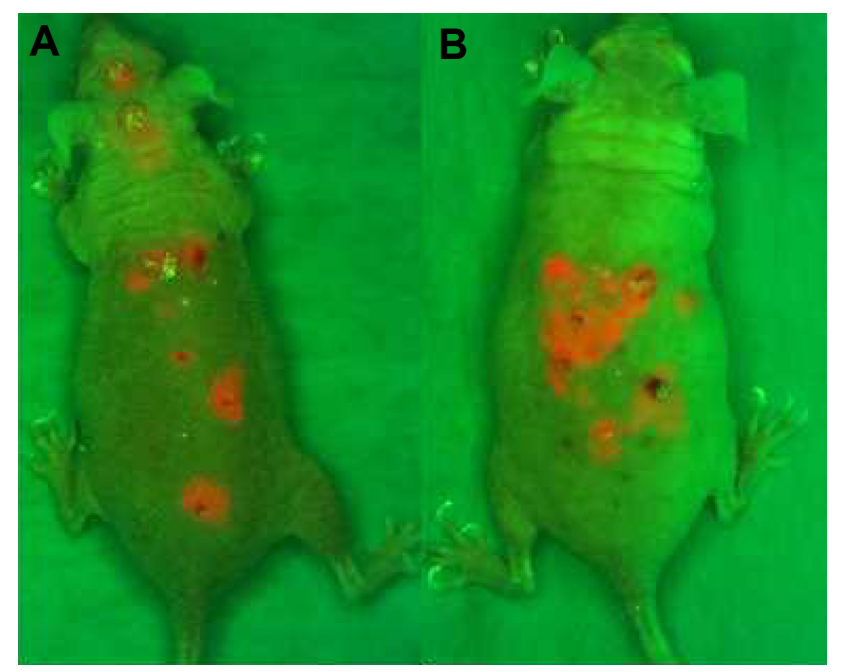

Figure 3 PpIX fluorescence images obtained at 6 hours of incubation.

Notes: The ALA PLGA NP group (A) shows better PpIX localization than the $0.8 \%$ ALA group (B).

Abbreviations: PpIX, protoporphyrin IX; ALA, 5-aminolevulinic acid; PLGA, polylactic-co-glycolic acid; NP, nanoparticle.

\section{Antitumor effect of ALA PLGA NP PDT}

The follow-up of tumor size showed that similar to the control group, tumors in the NP group continued to grow, with the volume increasing up to four times. The combined volume of ten tumors examined in the ALA PDT group was similar to that before treatment and continued to grow, and the volume increased up to three times after 6 weeks. In contrast, multiple sessions of ALA PLGA NP PDT significantly inhibited tumor growth. In the ALA PLGA NP PDT group, a week after the second treatment, tumor sizes were significantly reduced $(P<0.0001)$. A week after the fourth treatment, tumor sizes were significantly decreased compared with a week after the second treatment $(P<0.01)$. On average, the volume of tumors reduced $68 \% 2$ weeks after four treatments. Some smaller tumors $\left(<35 \mathrm{~mm}^{3}\right)$ were completely removed after one treatment. Among ten tumors examined, one tumor of $71.85 \mathrm{~mm}^{3}$ was removed after one session and one of $64.25 \mathrm{~mm}^{3}$ after three sessions (Figure 4).

\section{ALA PLGA NP PDT enhances antitumor immunity against SCC}

Both ALA PLGA NP PDT and ALA PDT induced the overexpression of $\mathrm{CD} 4^{+}$and $\mathrm{CD} 8^{+} \mathrm{T}$ cells $(P<0.01)$, but the number of $\mathrm{CD}^{+}$and $\mathrm{CD} 8^{+} \mathrm{T}$ cells of the ALA PLGA NP PDT group was higher than that of the ALA PDT group $(P<0.01)$ (Figure 5 and 6). However, the ratios of $\mathrm{CD} 4^{+}: \mathrm{CD} 8^{+}$positive cells of the ALA PLGA NP PDT group as well as the ALA PDT group were close to one (data not shown). For the control group and NP group, there was no noticeable change in the number of $\mathrm{CD} 4^{+}$and $\mathrm{CD} 8^{+} \mathrm{T}$ cells before and after treatment.

\section{Effect of microneedling}

In situ fluorescence examination showed that the PpIX production of the ALA PLGA NP group and the microneedle combination group was higher than the ALA group $(P<0.05)$. Although the use of microneedles slightly increased PpIX production, statistical analysis indicated that there was no significant difference $(P>0.05)$ (Figure 2). Transmission electron microscopy examination showed that most of the ALA PLGA NPs remained intact in treated tumors (Figure 7A and D). A large number of PLGA NPs were distributed in the cytoplasm of tumor cells (Figure 7B, C, $E$, and F). Moreover, the number of ALA PLGA NPs in the tumor cells of the combination group was threefold higher than that in the ALA PLGA NP group (30.5 \pm 1.5 versus $11.5 \pm 4.5)$. 

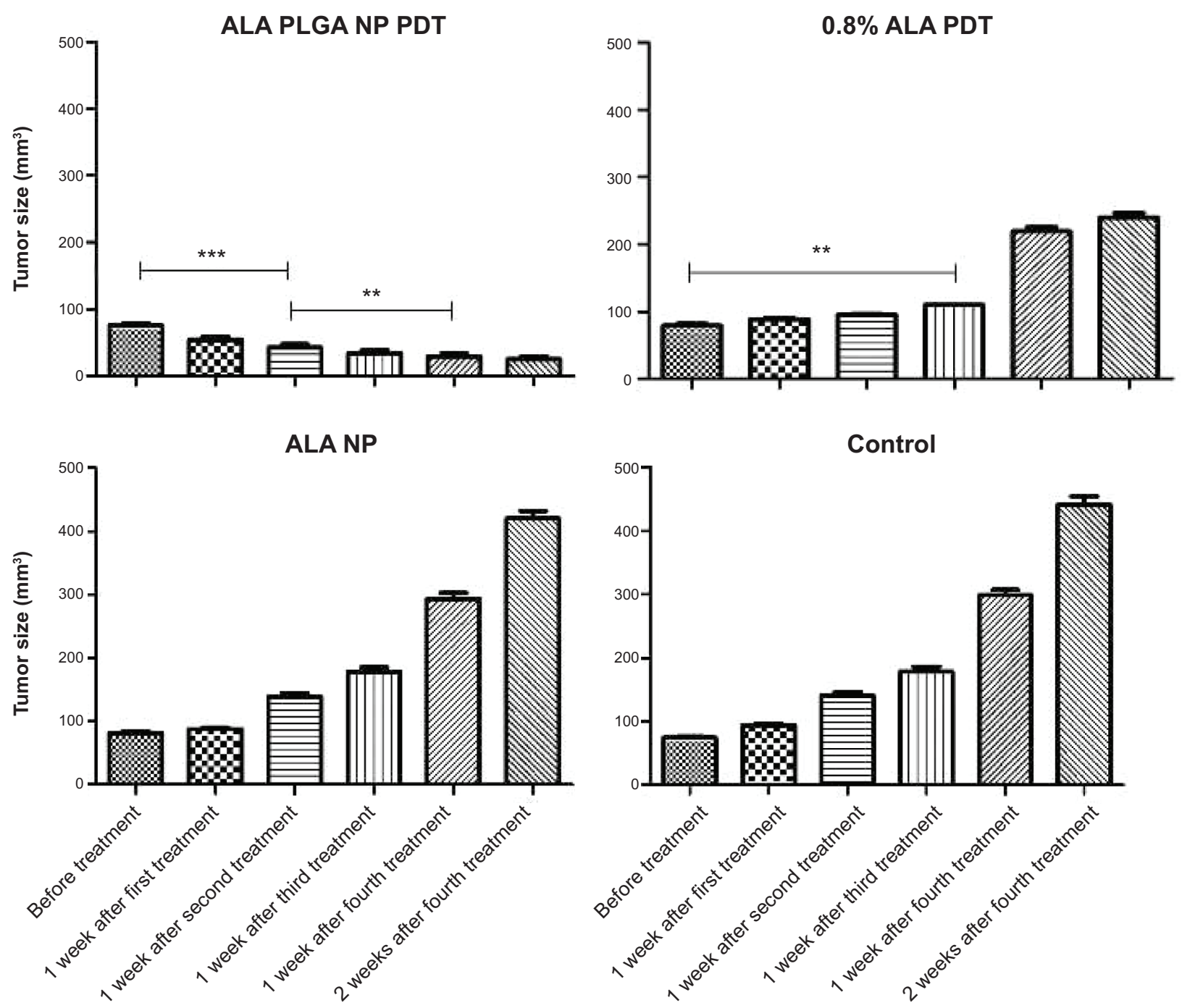

Figure 4 Measurement of tumor size.

Notes: $* * P<0.01$; $* * * P<0.0001$. Data presented as means \pm standard deviation from at least three independent experiments.

Abbreviations: ALA, 5-aminolevulinic acid; PLGA, polylactic-co-glycolic acid; NP, nanoparticle; PDT, photodynamic therapy.

\section{Discussion}

PDT involves the accumulation of photosensitizer in diseased tissues, followed by illumination of the disease site with light of an appropriate wavelength to induce photochemical reactions that result in tissue destruction. Topical PDT is becoming an established treatment for actinic keratosis, Bowen's disease, and superficial basal cell carcinoma. ${ }^{11-13}$

ALA is a prodrug of porphyrins and widely used in topical PDT around the world. ${ }^{14-16}$ However, the insufficient local bioavailability can limit the effectiveness of ALA-PDT. Lee et al showed that insoluble PpIX encapsulated in chitosan NPs had excellent photodynamic activity and were able to effectively induce the regression of skin tumors in mice. ${ }^{17}$ da Silva et al reported that delivery systems based on PLGA NPs had the potential to increase PpIX penetration into deep layers in the skin. ${ }^{6}$ PLGA has been used as a nanocarrier for many bioactive molecules (eg, drugs, peptides, proteins, deoxyribonucleic acid, and oligonucleotides), owing to its low toxicity, good biocompatibility, and US Food and Drug Administration approval status. ${ }^{18}$ The advantages of a PLGA NP-delivery system might include 1) selective accumulation of therapeutic concentrations of PLGA NPs within the diseased tissues, with little or no uptake by nontarget cells, 2) effective penetration through the stratum corneum, and 3) improvement in the vehicle/stratum corneum partition coefficient $(\mathrm{Kv} / \mathrm{sc}){ }^{19-23}$

A modified double-emulsion solvent-evaporation method was used to entrap ALA in PLGA NPs in this study, which resulted in a relatively good particle-size distribution $(65.6 \pm 26 \mathrm{~nm})$ and high encapsulation efficiency 

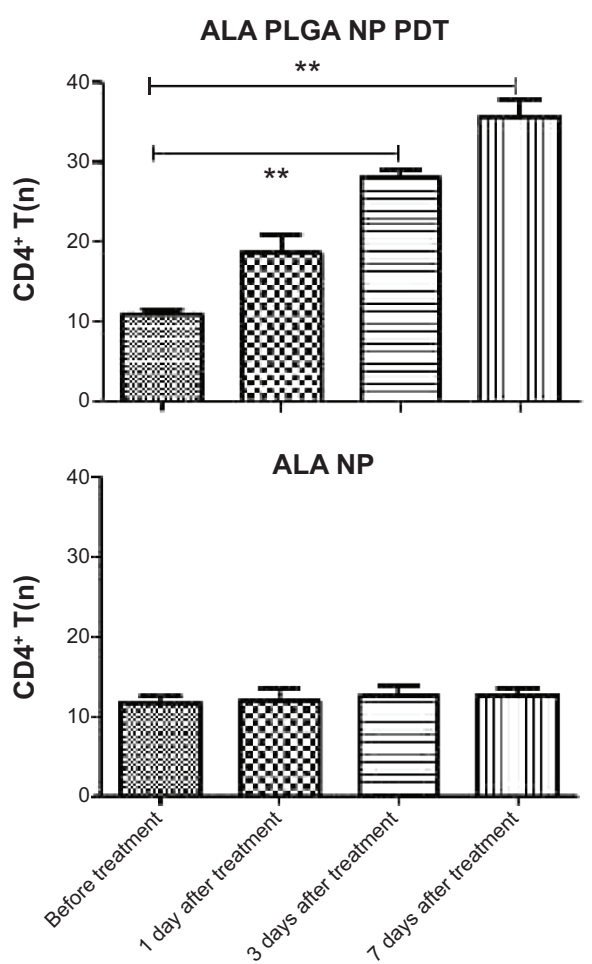

$0.8 \%$ ALA PDT

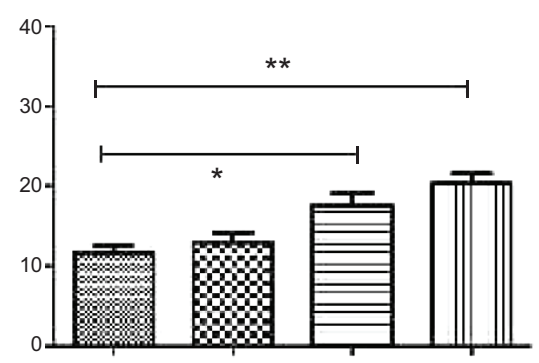

Control

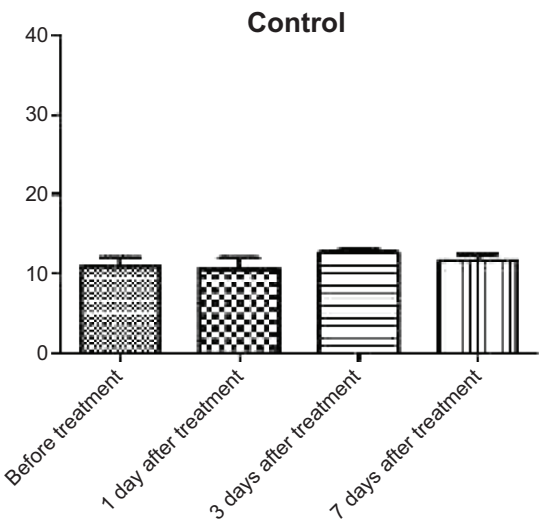

Figure 5 Measurement of $\mathrm{CD} 4^{+}$cells. Positive cell number/field/slice (mean \pm standard deviation, $n=3$ ).

Notes: $* P<0.05 ; * * P<0.01$.

Abbreviations: ALA, 5-aminolevulinic acid; PLGA, polylactic-co-glycolic acid; NP, nanoparticle; PDT, photodynamic therapy.

ALA NP PDT
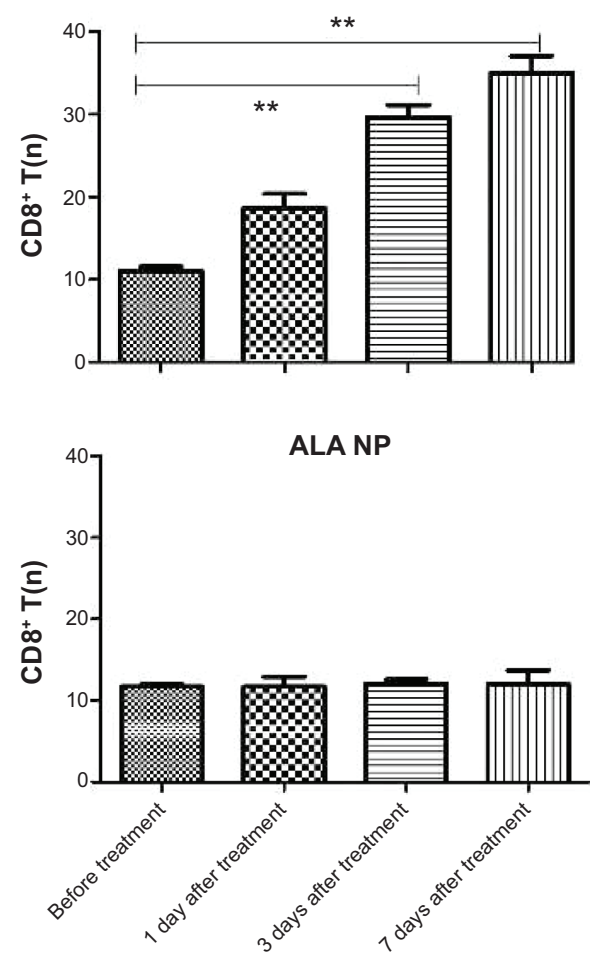

$0.8 \%$ ALA PDT

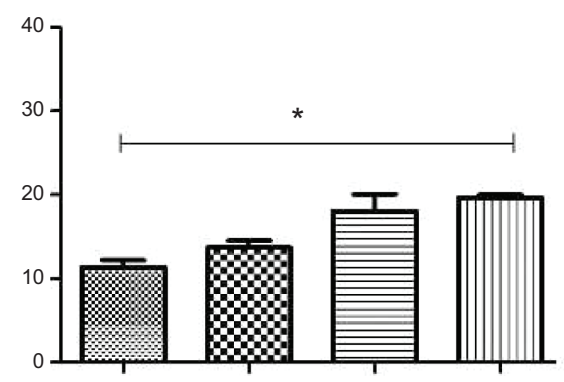

Control

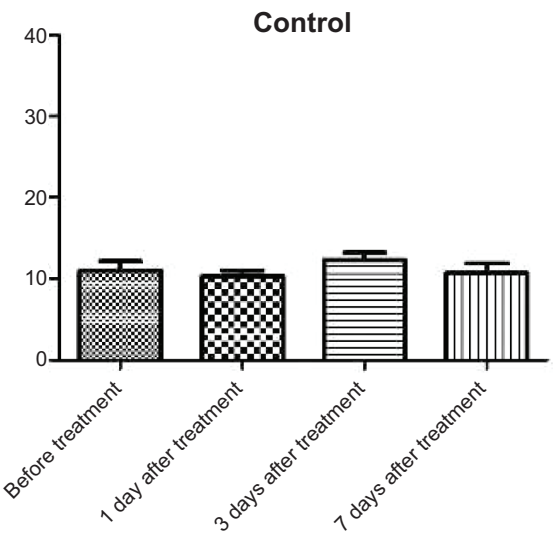

Figure 6 Measurement of CD8 ${ }^{+}$cells. Positive cell number/field/slice (mean \pm standard deviation, $n=3$ ).

Notes: $* P<0.05 ; * * P<0.01$.

Abbreviations: ALA, 5-aminolevulinic acid; NP, nanoparticle; PDT, photodynamic therapy. 

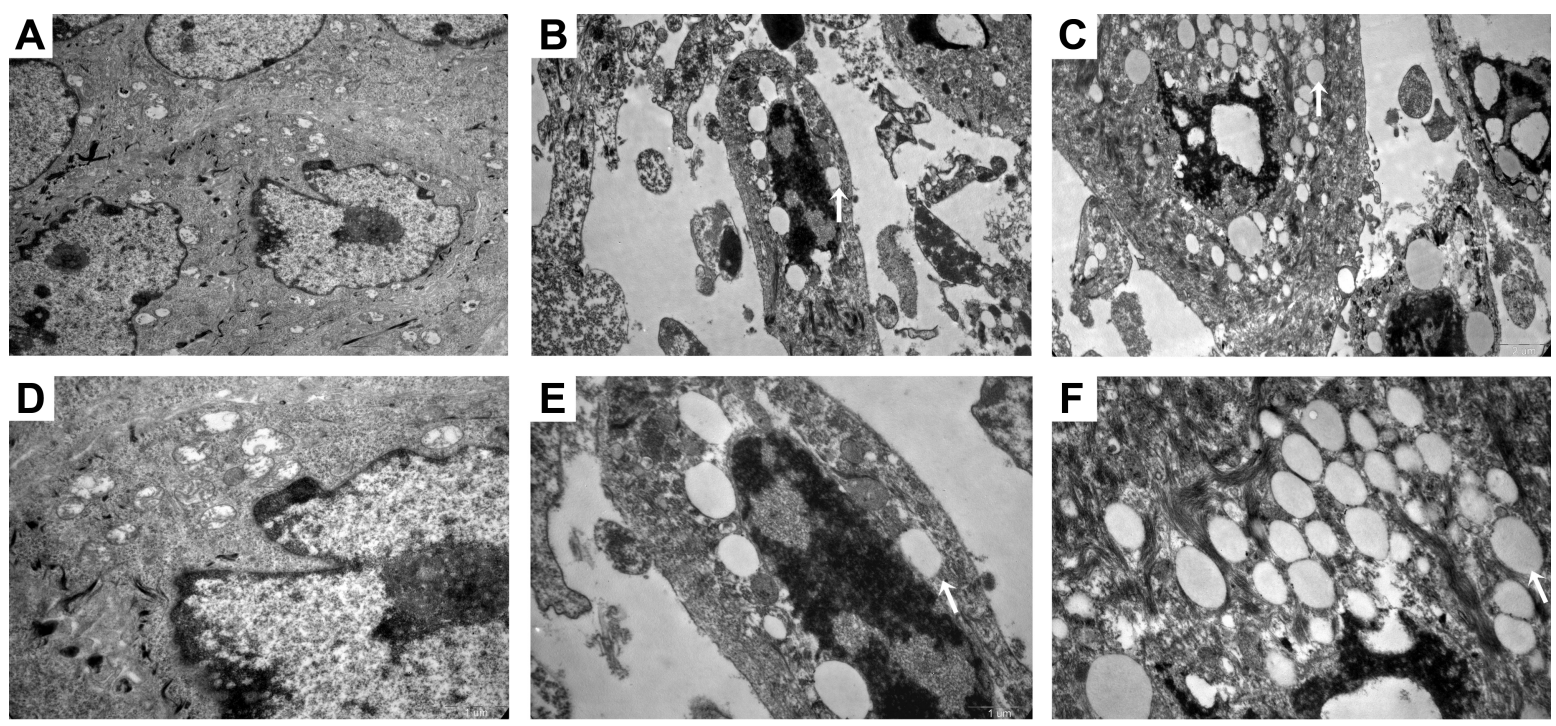

Figure 7 Electron microscopic examination of cellular localization of ALA PLGA NPs.

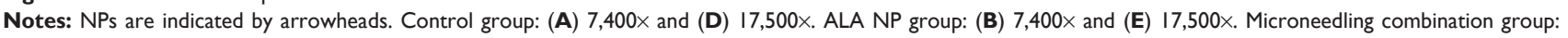
(C) 7,400x and (F) 17,500x.

Abbreviations: ALA, 5-aminolevulinic acid; PLGA, polylactic-co-glycolic acid; NPs, nanoparticles.

$(65.8 \% \pm 7.2 \%)$, although small hydrophilic molecules are not considered ideal candidates for PLGA NPs. ${ }^{8}$

The examination of in situ PpIX-fluorescence kinetics showed that SCCs incubated with ALA PLGA NPs generated higher levels of PpIX than those incubated with free ALA of the same concentration (Figure 2). This is possibly because free ALA might be directly dissolved in the extracellular compartment and decompose, whereas PLGA NPs could protect ALA from such degradation. ${ }^{8}$ Fluorescence images showed that the PpIX production and accumulation of the ALA PLGA NP group were stronger, more confined to the tumor site, and had weaker fluorescence intensity of surrounding tumor, compared to the ALA group (Figures 2 and 3). The low tissue penetrability of free ALA might limit its application for deep cancers. In addition, enhanced tumor-specific targeting behavior might occur through the enhanced permeation and retention effect. The enhanced tumor accumulation of ALA PLGA NPs could further improve therapeutic efficacy against SCC tumors. Indeed, the tumor volume of the ALA PLGA NP PDT group was significantly smaller than that of the ALA PDT group, although the latter showed a slight decrease in tumor volume compared to the control group (Figure 4). Since ALA PLGA NP PDT required lower doses of ALA, this might reduce the side effects and cost.

ALA PLGA NP PDT also reduced tumor recurrence. Immunohistochemical examination showed that the numbers of $\mathrm{CD}^{+} \mathrm{T}$ cells and $\mathrm{CD} 8^{+} \mathrm{T}$ cells increased gradually after ALA PLGA NP PDT (Figures 5 and 6), which suggests the elicitation of specific cellular immunity against SCCs. ALA PLGA NP PDT might promote $T$ cell activation and enhance adaptive antitumor immunity that might contribute to the prevention of recurrence. Since the number of $\mathrm{CD}^{+}$ cells and $\mathrm{CD} 8^{+}$cells in the ALA PLGA NP PDT group was higher than that in the ALA PDT group, this might suggest the possible synergistic effect of NPs and PDT in triggering T cell accumulation and activation. A study by Hinrichs et al indicated that naïve $\mathrm{CD} 8^{+}$cytotoxic $\mathrm{T}$ cells in humans and mice are the predominant cells mediating tumor destruction. ${ }^{24}$ Possibly through the secretion of $\mathrm{TNF} \alpha, \mathrm{CD}^{+}$cells may activate $\mathrm{CD} 8^{+}$cells, which could in turn exert a cytotoxic action on SCC cells. ${ }^{3}$ In recent years, more attention has been paid to $\mathrm{CD}^{+}{ }^{+} \mathrm{T}$ cells and their role in anti-infection and antitumor activity. ${ }^{25,26}$ Studies indicate that $\mathrm{CD} 4^{+} \mathrm{T}$ cells are required for the generation and maintenance of $\mathrm{CD} 8^{+}$memory $\mathrm{T}$ cells and also promote their survival. ${ }^{27}$

As shown by Henry et al in $1998,{ }^{28}$ microneedles with the length of only a few hundred microns are able to effectively penetrate the stratum corneum, which has a thickness of 10-20 mm. Compared to classical hypodermic needles, microneedles have the advantage of being relatively painless, although they are inserted at least into the epidermis and sometimes deeper into the superficial dermis, where nerves are present. Most likely, their small size reduces the odds of encountering a nerve or of stimulating it to produce a painful sensation. ${ }^{28,29}$ Vandervoort and Ludwig described a combination technique for enhancing PDT efficacy for treating photoaging. ${ }^{30}$ The initial step of the combination 
technique employs a microneedle roller to enhance topical penetration and delivery of ALA.

We also investigated the effect of microneedles on ALA delivery. PpIX-fluorescence kinetics results (Figure 2) showed that ALA PLGA NP-mediated PpIX fluorescence in tumors increased with the pretreatment of tumors with microneedling. A large number of spherical ALA PLGA NPs were seen in the cytoplasm of tumor cells (Figure 7). After 3 hours of incubation, the majority of ALA PLGA NPs in the cytoplasm were maintained spherical. The number of ALA PLGA NPs in the tumor tissue pretreated with microneedling was significantly higher. The microneedling might be useful for delivering ALA to thicker lesions.

In conclusion, ALA PLGA NPs can enhance in vivo ALA PDT efficiency and induce antitumor immune responses in SCC tumor-bearing mice. A combination of microneedling might be a promising method to further improve transdermal delivery of ALA PLGA NPs. The use of PLGA NPs and microneedling in topical ALA PDT deserves further investigation.

\section{Acknowledgments}

This work was supported by the National Natural Science Foundation of China (81272990), the Talent Training Project of Shanghai Health Institution of Shanghai Municipal Health Bureau (XBR2011059), Key Project of Shanghai (13JC1405101), and the Class A Key Project of Shanghai Municipal Health Bureau (20124034). The authors would like to thank Li Yang and Yazhen Wang of the National Pharmaceutical Engineering Research Center for assisting with NP preparation.

\section{Disclosure}

The authors report no conflicts of interest in this work.

\section{References}

1. Wang XL, Wang HW, Guo MX, Xu SZ. Treatment of skin cancer and pre-cancer using topical ALA-PDT - a single hospital experience. Photodiagnosis Photodyn Ther. 2008;5(2):127-133.

2. Wang XL, Wang HW, Guo MX, Yu YF. [Treatment of skin cancer and pre-cancer using topical ALA-PDT]. Chin J Dermatol. 2006;39(3): 137-139. Chinese.

3. Wang H, Li J, Lv T, Tu Q, Huang Z, Wang X. Therapeutic and immune effects of 5-aminolevulinic acid photodynamic therapy on UVB-induced squamous cell carcinomas in hairless mice. Exp Dermatol. 2013;22(5): 362-363.

4. Araújo LM, Thomazine JA, Lopez RF. Development of microemulsions to topically deliver 5-aminolevulinic acid in photodynamic therapy. Eur J Pharm Biopharm. 2010;75(1):48-55.

5. Ding H, Sumer BD, Kessinger CW, et al. Nanoscopic micelle delivery improves the photophysical properties and efficacy of photodynamic therapy of protoporphyrin IX. J Control Release. 2011;151(3): 271-277.
6. da Silva CL, Del Ciampo JO, Rossetti FC, Bentley MV, Pierre MB. Improved in vitro and in vivo cutaneous delivery of protoporphyrin IX from PLGA-based nanoparticles. Photochem Photobiol. 2013;89(5): 1176-1184.

7. Lee KD, Jeong YI, Kim da H, Lim GT, Choi KC. Cisplatin-incorporated nanoparticles of poly(acrylic acid-co-methyl methacrylate) copolymer. Int J Nanomedicine. 2013;8:2835-2845.

8. Shi L, Wang X, Zhao F, et al. In vitro evaluation of 5-aminolevulinic acid (ALA) loaded PLGA nanoparticles. Int J Nanomedicine. 2013; 8:2669-2676.

9. Lv T, Huang ZF, Wang HW, et al. Evaluation of collagen alteration after topical photodynamic therapy (PDT) using second harmonic generation (SHG) microscopy - in vivo study in a mouse model. Photodiagnosis Photodyn Ther. 2012;9(2):164-169.

10. Ackerman AB, Mones JM. Solar (actinic) keratosis is squamous cell carcinoma. Br J Dermatol. 2006;155(1):9-22.

11. Clementoni MT, B-Roscher M, Munavalli GS. Photodynamic photorejuvenation of the face with a combination of microneedling, red light, and broadband pulsed light. Lasers Surg Med. 2010;42(2):150-159.

12. Braathen LR, Szeimies RM, Basset-Seguin N, et al. Guidelines on the use of photodynamic therapy for nonmelanoma skin cancer: an international consensus. International Society for Photodynamic Therapy in Dermatology, 2005. J Am Acad Dermatol. 2007;56(1): 125-143.

13. Morton CA, McKenna KE, Rhodes LE. Guidelines for topical photodynamic therapy: update. Br J Dermatol. 2008;159(6):1245-1266.

14. Buinauskaite E, Maciulaitis R, Buinauskiene J, Valiukeviciene S. Topical photodynamic therapy of actinic keratoses with 5-aminolevulinic acid: randomized controlled trial with six months follow-up. J Dermatolog Treat. 2014;25(6):519-522.

15. Ma L, Xiang LH, Yu B, et al. Low-dose topical 5-aminolevulinic acid photodynamic therapy in the treatment of different severity of acne vulgaris. Photodiagnosis Photodyn Ther. 2013;10(4):583-590.

16. Sidoroff A, Thaler $P$. Taking treatment decisions in non-melanoma skin cancer - the place for topical photodynamic therapy (PDT). Photodiagnosis Photodyn Ther. 2010;7(1):24-32.

17. Lee SJ, Park K, Oh YK, et al. Tumor specificity and therapeutic efficacy of photosensitizer-encapsulated glycol chitosan-based nanoparticles in tumor-bearing mice. Biomaterials. 2009;30(15):2929-2939.

18. Lu JM, Wang X, Marin-Muller C, et al. Current advances in research and clinical applications of PLGA-based nanotechnology. Expert Rev Mol Diagn. 2009;9(4):325-341.

19. Miyazaki S, Takahashi A, Kubo W, Bachynsky J, Löebenberg R. Poly n-butylcyanoacrylate (PNBCA) nanocapsules as a carrier for NSAIDs: in vitro release and in vivo skin penetration. J Pharm Pharm Sci. 2003; 6(2):238-245.

20. Alvarez-Román R, Naik A, Kalia YN, Guy RH, Fessi H. Enhancement of topical delivery from biodegradable nanoparticles. Pharm Res. 2004;21(10):1818-1825.

21. Alvarez-Román R, Naik A, Kalia YN, Guy RH, Fessi H. Skin penetration and distribution of polymeric nanoparticles. J Control Release. 2004;99(1):53-62.

22. Luengo J, Weiss B, Schneider M, et al. Influence of nanoencapsulation on human skin transport of flufenamic acid. Skin Pharmacol Physiol. 2006;19(4):190-197.

23. Lademann J, Richter H, Teichmann A, et al. Nanoparticles - an efficient carrier for drug delivery into the hair follicles. Eur J Pharm Biopharm. 2007;66(2):159-164.

24. Hinrichs CS, Borman ZA, Cassard L, et al. Adoptively transferred effector cells derived from naive rather than central memory CD8+ T cells mediate superior antitumor immunity. Proc Natl Acad Sci U S A. 2009;106(41):17469-17474.

25. Brown DM. Cytolytic CD4 cells: direct mediators in infectious disease and malignancy. Cell Immunol. 2010;262(2):89-95.

26. Perez-Diez A, Joncker NT, et al. CD4 cells can be more efficient at tumor rejection than CD8 cells. Blood. 2007;109(12): 5346-5354. 
27. Janssen EM, Droin NM, Lemmens EE, et al. CD4+ T-cell help controls CD8+ T-cell memory via TRAIL-mediated activation-induced cell death. Nature. 2005;434(7029):88-93.

28. Henry S, McAllister DV, Allen MG, Prausnitz MR. Microfabricated microneedles: a novel approach to transdermal drug delivery.J Pharm Sci. 1998;87(8):922-925.
29. Kaushik S, Hord AH, Denson DD, et al. Lack of pain associated with microfabricated microneedles. Anesth Analg. 2001;92(2):502-504.

30. Vandervoort J, Ludwig A. Microneedles for transdermal drug delivery: a minireview. Front Biosci. 2008;13:1711-1715.

International Journal of Nanomedicine

\section{Publish your work in this journal}

The International Journal of Nanomedicine is an international, peerreviewed journal focusing on the application of nanotechnology in diagnostics, therapeutics, and drug delivery systems throughout the biomedical field. This journal is indexed on PubMed Central, MedLine, CAS, SciSearch $®$, Current Contents $® /$ Clinical Medicine,
Journal Citation Reports/Science Edition, EMBase, Scopus and the Elsevier Bibliographic databases. The manuscript management system is completely online and includes a very quick and fair peer-review system, which is all easy to use. Visit http://www.dovepress.com/ testimonials.php to read real quotes from published authors.

Submit your manuscript here: http://www.dovepress.com/international-journal-of-nanomedicine-journal 